\title{
Presiones sobre la conservación asociadas al uso de la tierra en las ecorregiones terrestres de la Argentina
}

\author{
A. Sofía Nanni ${ }^{1, \otimes}$; María Piquer-RodrígueZ ${ }^{1,2,3} ;$ Daniela RodrígueZ ${ }^{4,5} ;$ Mauricio \\ Nuñez-Regueiro ${ }^{67} ;$; M. Eugenia Periago ${ }^{8}$; Sebastián Aguiar ${ }^{9}$; Sebastián A. Ballari ${ }^{10}$; \\ Cecilia Blundo ${ }^{1}$; Enrique Derlindati ${ }^{6,11}$; Yamil Di Blanco ${ }^{12}$; Ana Eljall ${ }^{8}$; Ricardo \\ H. Grau'; Lorena Herrera ${ }^{13}$; Alejandro Huertas Herrera ${ }^{14}$; Andrea E. Izquierdo'; \\ Julián Lescano ${ }^{15}$; Leandro Macchi ${ }^{1}$; Flavia Mazzini ${ }^{16}$; Mayra Milkovic ${ }^{6}$; Lia

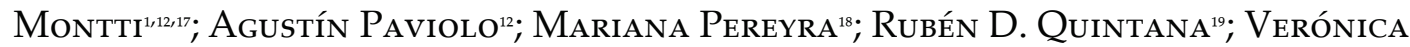 \\ Quiroga $^{1215} ;$ Daniel Renison ${ }^{20}$; Mario Santos BeAdE ${ }^{21}$; Alejandro SchaAF ${ }^{22}$ \& N. \\ IGNACIO GASPARRI ${ }^{1}$ \\ ${ }^{1}$ Instituto de Ecología Regional (UNT-CONICET), Tucumán. **
}

\begin{abstract}
Resumen. Por su continuidad espacial y su relativa homogeneidad ecológica y biofísica, las ecorregiones son un nivel de organización apropiado para discutir y planificar iniciativas de conservación del ambiente a escala regional y nacional. Las interacciones complejas entre las características ecológicas de las ecorregiones, los cambios socioeconómicos locales y globales, y los cambios en el uso de la tierra ocasionan que las amenazas y la oportunidades para conservar dentro y entre ecorregiones se distribuyan de manera heterogénea. En este artículo realizamos un diagnóstico actualizado de las presiones de conservación asociadas al uso de la tierra en las distintas ecorregiones terrestres de la Argentina. Entendemos por presiones a las actividades antrópicas que modifican el estado de los recursos naturales e impactan sobre su funcionamiento. Luego de identificar las principales presiones por ecorregión realizamos un análisis de agrupamiento para identificar grupos de ecorregiones en función de la similitud de sus presiones. La ganadería comercial y la agricultura estuvieron dentro de las principales presiones asociadas al uso de la tierra en ocho y siete de las ecorregiones terrestres de la Argentina, respectivamente. Otras presiones de incidencia más específicas fueron muy relevantes en algunas ecorregiones, en especial en aquellas con limitantes para las actividades agropecuarias orientadas a los mercados globales. Identificamos cuatro grupos en función de la incidencia de las presiones asociadas al uso de la tierra: 1) Monte, Puna y Altos Andes; 2) Bosques Patagónicos; 3) Selva Paranaense y Selva de las Yungas, y 4) Campos y Malezales, Chaco Húmedo, Chaco Seco, Delta e Islas del Paraná, Espinal, Esteros del Iberá, Estepa Patagónica y Pampa. Este agrupamiento por presiones comunes de ecorregiones distantes en el espacio contribuiría a organizar geográficamente políticas de gestión de los recursos naturales integradas a nivel regional.
\end{abstract}

[Palabras clave: cambios en el uso de la tierra, conservación de la naturaleza, características ambientales, agricultura, ganadería comercial, usos antrópicos]

\begin{abstract}
Aвstract. Threats on conservation associated to land-use in the terrestrial ecoregions of Argentina. Ecoregions are a suitable organization level for the discussion and planning of conservation initiatives at a regional level, due to their spatial continuity and ecological and biophysical homogeneity. The complex interactions between ecological characteristics of ecoregions, local and global socioeconomic changes and land-use change determine that threats and opportunities for conservation within and among ecoregions are unequally distributed. In this article, we performed an updated diagnosis of the main conservation issues associated with land-use change in the terrestrial ecoregions of Argentina. Also, in each ecoregion we identified the main conservation threats derived from land-use change (defined as the anthropic activities that modify the state of natural resources, impacting on their functioning) and organized ecoregions in groups according to their similarity in the incidence of the respective threats, through a cluster analysis. Commercial livestock production and agriculture were among the main threats associated with land-use change in eight and seven terrestrial ecoregions of Argentina, respectively. Other more local threats were also relevant in certain ecoregions, especially for those that are unsuitable for intensive land-uses. We identified four groups according to the incidence of the threats: 1) Monte, Puna and Altos Andes; 2) Bosques Patagónicos; 3) Selva Paranaense and Selva de las Yungas, and 4) Campos y Malezales, Chaco Húmedo, Chaco Seco, Delta e Islas del Paraná, Espinal, Esteros del Iberá, Estepa Patagónica and Pampa. Such grouping of distant ecoregions by common threats would contribute to spatially organize integrated natural resource management policies that go beyond administrative boundaries.
\end{abstract}

[Keywords: land-use change, nature conservation, environmental characteristics, agriculture, commercial livestock production, anthropic uses]

Editor asociado: Germán Baldi

$\bar{\triangle}$ sofiananni@gmail.com
Recibido: 31 de Octubre de 2019

Aceptado: 27 de Abril de 2020 
${ }^{* 2}$ Latinamerika-Institut, Frei Univesität Berlin, Alemania. ${ }^{3}$ Geography Department, Humboldt Universität zu Berlin. ${ }^{4}$ IADIZA (CONICET), Mendoza. ${ }^{5}$ FCA-UNCuyo, Mendoza. ${ }^{6}$ IBIGEO (CONICET), Universidad Nacional de Salta. ${ }^{7}$ Universidad Católica de Salta, Salta. ${ }^{8}$ Fundación Vida Silvestre Argentina, Buenos Aires. ${ }^{9}$ Laboratorio de Análisis Regional y Teledetección (IFEVA-CONICET), FAUBA, Buenos Aires. ${ }^{10}$ Parque Nacional Nahuel Huapi. CENAC (APN/CONICET), Río Negro. ${ }^{11}$ Facultad de Ciencias Naturales, Universidad Nacional de Salta. ${ }^{12}$ IBS (CONICET-UNaM) y Centro de Investigaciones del Bosque Atlántico (CeIBA), Misiones. ${ }^{13}$ Grupo de Estudios en Agroecosistemas y Paisajes Rurales, Facultad de Ciencias Agrarias, Universidad de Mar del Plata, Buenos Aires. ${ }^{14}$ Centro Austral de Investigaciones Científicas (CADICCONICET), Tierra del Fuego. ${ }^{15}$ Instituto de Diversidad y Ecología Animal y Centro de Zoología Aplicada, UNC-CONICET, Córdoba. ${ }^{16}$ Centro de Estudios Territoriales Ambientales y Sociales, Universidad Nacional de Jujuy, Jujuy. ${ }^{17}$ Instituto de Investigaciones Marinas y Costeras (UNMdP-CONICET), Instituto de Geología de Costas y del Cuaternario (UNMdPCIC), Mar del Plata, Buenos Aires. ${ }^{18}$ Instituto Multidisciplinario de Biología Vegetal (CONICET), Córdoba. ${ }^{19}$ Instituto de Investigación e Ingeniería Ambiental (IIIA) CONICET-Universidad Nacional de San Martín, Buenos Aires. ${ }^{20}$ Centro de Ecología y Recursos Naturales Renovables (UNC-CONICET), Córdoba. ${ }^{21}$ Parque Nacional Campos del Tuyú (APN). ${ }^{22}$ INECOA (UNJu-CONICET), Jujuy.

\section{INTRODUCCIÓN}

Como ciencia, la biología de la conservación evolucionó de manera vertiginosa en el último siglo, junto con el creciente conocimiento y la percepción de la influencia de las actividades antrópicas sobre el ambiente (Menzel and Teng 2010; Mace 2014). En sus inicios, a mediados del siglo XIX, el paradigma dominante para la investigación en el área de la conservación era el de incompatibilidad entre la preservación de la naturaleza y las actividades humanas. Esto orientó a las estrategias de conservación de la naturaleza hacia identificar sitios prístinos y establecer reservas biológicas en áreas alejadas de centros poblados o con bajos niveles de intervención humana (Edwards and Abivardi 1998; Baldi et al. 2019). Durante las últimas décadas del siglo $\mathrm{XX}$ se empezaron a aplicar nuevos marcos conceptuales y enfoques metodológicos, como el de los servicios ecosistémicos (Costanza et al. 1997; Daily 1997) y los sistemas socioecológicos (Ostrom 2007). Estos nuevos enfoques permitieron el reconocimiento del vínculo y la integración entre sistemas sociales y naturales. De esta manera, los estudios en ecología y conservación empezaron a centrarse de manera progresiva en las consecuencias de los cambios antrópicos sobre los sistemas naturales (Zimmerer 2006).

La ciencia del uso del territorio (land science) emergió en el mencionado contexto de integración entre sistemas sociales y naturales, e incluye especialmente la dimensión geográfica. Esta ciencia busca comprender la dinámica de los cambios en el uso de la tierra y está muy asociada al concepto de sustentabilidad y al balance entre la conservación de la naturaleza y las actividades antrópicas (Turner et al. 2007; Verbourg et al. 2015). Al reconocerse los cambios en el uso de la tierra como la principal presión para la conservación de la naturaleza (Crossman et al. 2013; Díaz et al. 2019), las agendas prioritarias de investigación de la ciencia del uso del territorio y de la biología de la conservación se fueron acercando de manera progresiva (Hansen et al. 2012). Incluso, en la actualidad, la conservación del ambiente se encuentra entre los principales ámbitos internacionales de toma de decisiones, lo que se refleja en el establecimiento de más de 140 evaluaciones ambientales globales en las últimas décadas (Jabbour and Flachsland 2014). Entre las planificaciones de más impacto en la toma de decisiones se destacan la Agenda 2030 para el Desarrollo Sostenible de la ONU (United Nations 2015), la Plataforma Intergubernamental sobre Biodiversidad y Servicios Ecosistémicos para los Servicios Ecosistémicos (Brondizio et al. 2019) y el Panel Intergubernamental del Cambio Climático (IPCC) (IPCC 2014). Esta y otras iniciativas requieren entender las presiones sobre los ecosistemas asociadas a los cambios en el uso de la tierra, entendidas como "las actividades antrópicas vinculadas a la demanda de una necesidad que modifican el estado de los recursos naturales, causando una serie de impactos sobre su funcionamiento" (Kristensen et al. 2004).

Por su tamaño, su coherencia espacial y su relativa homogeneidad ecológica y biofísica, las ecorregiones son un nivel de organización biológica apropiado para analizar, evaluar y planificar la conservación del ambiente a nivel regional y nacional (Burkart et al. 1999; Olson et al. 2001; Brown et al. 2006; Dinerstein et al. 2019). Para un análisis socioecológico, el concepto de ecorregiones está limitado por tener un origen en unidades territoriales de ecosistemas naturales (Cabrera 1976), en contraste con modelos más integrados de zonificación socioecológica (e.g., Ellis and Ramankutty 2008). Sin embargo, la coherencia de las ecorregiones como unidades de organización biogeográfica se ha validado en términos de las comunidades biológicas (Smith et al. 2018), y su utilidad como 
unidades territoriales para discutir y organizar iniciativas de conservación es, en general, aceptada en distintos ámbitos, incluyendo el académico y el político (e.g., Dinerstein et al. 2019). En relación con esto último, los objetivos de Aichi para conservación global de la biodiversidad, por ejemplo, establecen proteger $17 \%$ de los ecosistemas terrestres tomando las ecorregiones como referencia (CBD 2010).

En general, las características ambientales que definen los límites de las ecorregiones (e.g., clima, suelo) condicionan los usos de la tierra dentro de las mismas. Por esta razón, la caracterización de usos antrópicos también se puede basar en las ecorregiones como referencia. Sin embargo, hay aspectos en los que la distribución de los usos antrópicos y los ecosistemas naturales están condicionados por variables muy distintas. La pendiente del terreno, por ejemplo, es un factor clave para la distribución de la agricultura mecanizada (Aide et al. 2013), pero influye poco sobre la distribución de las comunidades naturales. La influencia de los procesos socioeconómicos y ambientales globales o distantes (e.g., comercio internacional, aumento de la demanda global de alimentos, del consumo per cápita, cambio climático) sobre los cambios en el uso de la tierra locales aumentó de forma exponencial durante las últimas décadas. (Meyfroidt et al. 2010). Estas fuerzas conductoras globales o distantes influyen sobre los agentes locales de cambio de uso de la tierra, y en muchos casos promueven altas tasas de pérdida de áreas naturales y sobreutilización de los recursos (Vallejos et al. 2019). Las interacciones complejas entre las características ecológicas de las ecorregiones, los cambios socioeconómicos locales y globales, y los cambios en el uso de la tierra ocasionan que las presiones para la conservación dentro y entre ecorregiones se distribuyan de manera heterogénea, y que varíen en el tiempo (e.g., Izquierdo and Grau 2009; Redo et al. 2012). Por ejemplo, mientras que la Pampa y el Espinal fueron, por excelencia, las ecorregiones de expansión de la frontera agrícola durante inicios del siglo XX (Viglizzo et al. 2001), la ecorregión del Chaco Seco permaneció como un área de usos ganadero extensivos y forestales. Sin embargo, los cambios tecnológicos de las últimas décadas y el acceso a mercados externos facilitaron la expansión agropecuaria hacia el Chaco Seco, una de las regiones con las tasas de deforestación más altas del mundo en la actualidad (Gasparri and Grau
2009; Baumann et al. 2017). A este proceso se lo conoce como la pampeanización del chaco (Morello et al. 2005).

La expansión agropecuaria representa una seria presión ya reconocida sobre la conservación de la naturaleza en la Argentina debido a la gran superficie implicada y a las diversas consecuencias socioambientales asociadas (Viglizzo et al. 2011). Sin embargo, otros tipos de usos de la tierra de incidencia más puntual influyen sobre la conservación de la naturaleza, en particular en ecorregiones cuyas características biofísicas dificultan la expansión agropecuaria. Algunos ejemplos son la expansión de la minería en la Puna, la cacería y otras formas de explotación de la fauna silvestre y recursos madereros en el Chaco Seco, la selva de Yungas y Selva Paranaense, o las invasiones biológicas en la Patagonia (Paviolo et al. 2009; Quiroga et al. 2014; Ballari et al. 2016; Izquierdo et al. 2015, 2018; Campanello et al. 2019; Politi and Rivera 2019).

La Argentina es el segundo país más extenso de Sudamérica $\left(2791810 \mathrm{~km}^{2}\right.$ de superficie continental), y por su ubicación latitudinal y su heterogeneidad topográfica presenta una gran diversidad biofísica y ecológica. En la actualidad, el país está subdividido en 18 ecorregiones, de las cuales 15 corresponden al área continental y tres al área marítima (Burkart et al. 1999). Las diferentes ecorregiones varían en sus niveles de protección y transformación (Tabla 1), lo que sumado a la enorme heterogeneidad ecológica promueve diferentes presiones ambientales en cada una de ellas. Por otro lado, algunos aspectos de la gobernanza de los recursos naturales dificultan un análisis integral de las presiones antrópicas sobre la conservación de la naturaleza. Por ejemplo, el artículo 14 de la Constitución Nacional Argentina determina que la soberanía de los recursos naturales es de cada provincia, por lo cual, la gestión y la legislación relacionada a los mismos se realiza a este nivel. Sin embargo, algunos ejemplos o procesos legislativos en el país muestran la necesidad de una mayor injerencia del gobierno nacional en la planificación del uso del territorio. Tal es el caso de la Ley Nacional № 26.331 de "Presupuestos Mínimos de Protección Ambiental de los Bosques Nativos" (conocida como "Ley de Bosques"), sancionada en 2007 por el Congreso Nacional, que exigió a las provincias que efectuaran un Ordenamiento Territorial de sus bosques nativos. Aunque la 
Ley de Bosques tuvo múltiples debilidades en cuanto a su efectividad y coordinación entre la autoridad nacional de aplicación y sus equivalentes provinciales, ilustra la necesidad de planificar el uso de la tierra a una escala que exceda a la provincial (Piquer et al. 2015; Aguiar et al. 2018). En este contexto, identificar presiones antrópicas comunes y relevantes a nivel de ecorregión y entre ecorregiones es valioso para definir políticas nacionales de conservación, teniendo en cuenta que los límites ambientales trascienden a los límites político-administrativos.

En el 2005 se realizó un diagnóstico ambiental de cada una de las ecorregiones del país, que incorpora aspectos sociales, económicos y políticos, locales y globales que afectan al ambiente (Brown et al. 2006). Sin embargo, las grandes transformaciones del paisaje experimentadas en la última década en el país (e.g., incremento del 35\% en la superficie cultivada entre 2000 y 2010; Volante et al. [2015]), requiere actualizar periódicamente este tipo de análisis. El único mapa de coberturas del suelo bajo un mismo protocolo para la totalidad del territorio argentino se realizó en 2007 (INTA 2009). Desde entonces, el Sistema Nacional de Bosques Nativos generó mapas de cambio de cobertura en las regiones forestales (Parque Chaqueño, Selva Tucumano-Boliviana, Selva Misionera y Bosques Andino-Patagónicos). Además, existe un monitoreo de cambio de cobertura (desmonte) para el Chaco Seco, generado por el sector académico desde 1976 a la actualidad (Vallejos et al. 2015). Teniendo en cuenta este contexto, en este artículo: a) realizamos un diagnóstico descriptivo de las presiones de conservación asociadas al uso de la tierra en las distintas ecorregiones terrestres de la Argentina en la actualidad; b) identificamos las principales presiones sobre la conservación asociadas a los usos de la tierra en cada ecorregión, a través de un método semicuantitativo de categorización; y c) identificamos grupos de ecorregiones en base a las similitudes en la incidencia de las respectivas presiones, por medio de un análisis de agrupamiento.

\section{Materiales y Métodos}

Este trabajo es el resultado de un encuentro presencial promovido por Global Land Programme, Oficina Nodal Latinoamericana, y financiado por la Fundación Rufford, que tuvo lugar en octubre de 2017 en la ciudad de Tucumán. Participaron 25 investigadores e investigadoras que trabajan en las ecorregiones terrestres de la Argentina (Burkart et al. 2009). Se priorizó que la persona invitada hubiera recibido algún subsidio de la Fundación Rufford en algún momento de su carrera. Sin embargo, debido a que no todas las ecorregiones estuvieron representadas por investigadores/as con financiamiento Rufford, para cumplir con el objetivo de alcance nacional se incluyeron también profesionales que trabajan en dichas ecorregiones aunque no hubieran recibido tal financiamiento. Así, se logró cierta representatividad entre las distintas ecorregiones e instituciones vinculadas a la ecología y uso del territorio en el país.

Elaboración de diagnósticos de presiones sobre la conservación asociadas al uso de la tierra en las distintas ecorregiones terrestres de la Argentina

Previo al taller presencial y de manera virtual se conformaron grupos de entre una y cuatro personas cuyas líneas de investigación se vincularan con la ecorregión asignada, para realizar documentos de síntesis (de hasta 1000 palabras) sobre la situación actual de cada ecorregión (Anexo 1). Estas revisiones tuvieron por objetivo identificar de manera explícita la influencia de distintos procesos asociados al uso de la tierra, y su vínculo con las presiones sobre la conservación de cada una de las 13 ecorregiones analizadas. Cabe destacar que por su similitud en características ecológicas y tendencias de uso de la tierra, las ecorregiones Puna y Altos Andes, Monte de Llanuras y Mesetas, y Monte de Sierras y Bolsones fueron tratadas en un mismo diagnóstico. Se utilizaron las siguientes preguntas orientativas a modo de guía: 1) ¿Cuáles son las principales presiones de conservación en la ecorregión asignada? y ¿Cuáles de estas presiones atraviesan otras ecorregiones, o corresponden a situaciones más específicas de esta ecorregión en particular? 2) ¿Cuáles son los usos de la tierra dominantes en la ecorregión asignada? 3) De los usos de la tierra mencionados, intentar identificar agentes causales, distinguiendo entre: a- agentes causales locales (i.e., provincial, nacional) o distantes (i.e., internacional) y b- procesos crónicos (operando desde antes del año 2000) o recientes (operando o incrementando de manera considerable luego del año 2000).

Estos documentos sirvieron de base para luego discutir y categorizar las presiones 
asociadas al uso de la tierra en las ecorregiones terrestres de la Argentina durante la reunión presencial. A su vez, constituyen documentos de síntesis y actualización de las presiones para la conservación asociadas al uso de la tierra en las respectivas ecorregiones (Anexo 1).

En cada una de las ecorregiones terrestres de la Argentina calculamos el porcentaje de superficie transformada, natural/seminatural y protegida, en el entorno de un sistema de información geográfica. Para calcular la superficie transformada utilizamos información a nivel nacional y a nivel global, y distinguimos entre superficie urbana y superficie cultivada. La información a nivel nacional consistió en el mapa de cobertura de suelo de la República Argentina, del año 2007, (escala 1:250000, y para zonas urbanas 1:100000) (INTA 2009). A esa información la actualizamos con otra proveniente de desmontes históricos 1976-2018 (Vallejos et al. 2015; SADyDS 2016). Esta información se encuentra disponible sólo para las ecorregiones boscosas subtropicales (i.e., Chaco Seco, Chaco Húmedo, Espinal, Selva Paranaense y Yungas) (escalas 1:10000 y 1:5000). Dentro de superficie cultivada y/o manejada se incluyen cultivos de especies arbóreas, cultivos de arbustos, cultivos de herbáceas, y áreas urbanas vegetadas (INTA 2009). Debido a su mayor resolución, consideramos la fuente nacional de información como primaria., pero al encontrarse disponible sólo para 2007, se complementó con información alternativa a nivel global. En este caso, la superficie urbana de cada ecorregión se calculó a partir de la capa GHS Settlement Model Grid de la base de datos Global Human Settlement, 2014 (Pesaresi et al. 2019). Esta capa clasifica niveles de urbanización (i.e., ciudades, pueblos y suburbios, áreas rurales) combinando información de superficie construida y densidad de población a distintas resoluciones (en este trabajo utilizamos una resolución espacial de $1 \mathrm{~km}$ ). Para calcular la superficie cultivada, utilizamos la base de datos Global Cropland Area Database, de superficie cultivada para el año 2015, a una resolución espacial de $30 \mathrm{~m}$ (Teluguntla et al. 2015; Zhong et al. 2017). En el caso de ambas bases de datos (global y nacional) consideramos como superficie natural/semi-natural al porcentaje restante de la superficie de cada ecorregión no transformada, si bien esta no está exenta de modificaciones antrópicas que pudieran no ser detectables a través del análisis espacial. Finalmente, calculamos la superficie protegida en base a WDPA (2019), una base de datos global de áreas protegidas marinas y terrestres, actualizada mes a mes, disponible en formato vectorial. De esta manera obtuvimos estimaciones del área total transformada por ecorregión a partir de dos fuentes alternativas de información, y una única estimación del porcentaje de cada ecorregión bajo protección (Tabla 1; Tabla A1).

Identificación y jerarquización de presiones asociadas al uso de la tierra en las ecorregiones terrestres de la Argentina

En una primera etapa, identificamos y categorizamos las presiones asociadas al uso de la tierra siguiendo la definición de presiones propuesta por el marco conceptual DPSIR (Driving-Forces-Pressures-State-ImpactsResponses) (Kristensen et al. 2004). Así, entendemos en este trabajo a las presiones como "las actividades antrópicas vinculadas a la demanda de una necesidad, que modifican el estado de los recursos naturales causando una serie de impactos sobre su funcionamiento" (Kristensen et al. 2004). Establecimos 11 presiones específicamente asociadas al uso antrópico de la tierra (excluyendo aquellas originadas por otros factores como cambio climático o desastres naturales) cuya incidencia consideramos relevante sobre los recursos naturales de las ecorregiones terrestres de la Argentina: 1) Extracción de Recursos Naturales no Renovables; 2) Cacería; 3) Aprovechamiento Forestal de Bosques Nativos; 4) Fuegos por Actividades Antrópicas; 5) Ganadería Comercial; 6) Turismo; 7) Plantaciones Forestales; 8) Agricultura; 9) Invasiones Biológicas; 10) Urbanización e Infraestructura, y 11) Ganadería de Subsistencia. Luego, cada grupo de trabajo cuantificó la influencia de cada presión en la ecorregión asignada aplicando un método semicuantitativo de jerarquización de presiones modificado del software Miradi v.4.3.1 (Miradi 2017). Este método requiere evaluar cuatro dimensiones de presiones: probabilidad de ocurrencia, extensión geográfica, severidad e irreversibilidad (ver definiciones de las cuatro dimensiones en Miradi 2017). Cada una de estas dimensiones permite una asignación categórica de mayor a menor incidencia (e.g., extensión geográfica cuenta con cinco categorías, desde "una muy pequeña parte del área geográfica" a "la totalidad del área geográfica"), que se convierte en una asignación numérica como proporción de 
Tabla 1. Porcentaje de cobertura modificada o transformada y natural/semi-natural en las distintas ecorregiones terrestres de la Argentina (recopilación generada por Fundación Vida Silvestre en base a INTA 2009[1], Monitoreo de desmontes realizado por LART, FAUBA, INTA y Redaf colección $7.0^{[2]}$, Monitoreo de bosques de UMSEF periodo 2006$2016^{[3]}$ ) y porcentaje de superficie protegida en cada ecorregión (recopilación generada por Fundación Vida Silvestre en base a WDPA ${ }^{[4]}$ agosto 2019 teniendo en consideración áreas protegidas terrestres nacionales y subnacionales).

Table 1. Percentage of modified and / or transformed land-cover, and natural/semi-natural land-cover in the terrestrial ecoregions of Argentina (compilation generated by Fundación Vida Silvestre based on INTA 2009 ${ }^{[1]}$, Deforestation monitoring by LART, FAUBA, INTA and Redaf collection $7.0{ }^{[2]}$, UMSEF Forest monitoring for 2006-2016 ${ }^{[3]}$ ) and percentage of protected area in each ecoregion (compilation generated by Fundación Vida Silvestre based on WDPA ${ }^{[4]}$ august 2019, considering national and subnational terrestrial protected areas).

\begin{tabular}{lccccc}
\hline $\begin{array}{l}\text { Ecorregión } \\
\text { (Burkart et al. 1999) }\end{array}$ & \multicolumn{3}{c}{ Modificado o transformado (\%) } & $\begin{array}{c}\text { Natural/semi- } \\
\text { natural (\%) }\end{array}$ & Protegido (\%) \\
& Total & Urbano & $\begin{array}{c}\text { Cultivado o } \\
\text { manejado }\end{array}$ & & \\
\hline Altos Andes & 0.15 & 0.00 & 0.15 & 99.85 & 23.36 \\
Bosques Patagónicos & 0.28 & 0.14 & 0.14 & 99.72 & 41.94 \\
Campos y Malezales & 20.84 & 0.47 & 20.37 & 79.16 & 1.31 \\
Chaco Húmedo & 14.64 & 0.14 & 14.50 & 85.36 & 1.31 \\
Chaco Seco & 20.13 & 0.12 & 20.01 & 79.87 & 4.78 \\
Delta e Islas del Paraná & 10.84 & 1.15 & 9.69 & 89.16 & 13.74 \\
Espinal & 48.64 & 0.29 & 48.35 & 51.36 & 1.58 \\
Estepa Patagónica & 0.32 & 0.04 & 0.28 & 99.68 & 6.24 \\
Esteros del Iberá & 7.02 & 0.08 & 6.94 & 92.98 & 28.08 \\
Monte de Llanuras y Mesetas & 3.78 & 0.27 & 3.51 & 96.22 & 3.19 \\
Monte de Sierras y Bolsones & 0.88 & 0.00 & 0.88 & 99.12 & 12.75 \\
Pampa & 78.02 & 0.88 & 77.14 & 21.98 & 2.66 \\
Puna & 0.09 & 0.00 & 0.09 & 99.91 & 20.24 \\
Selva Paranaense & 51.12 & 0.62 & 50.50 & 48.88 & 10.05 \\
Selva de las Yungas & 19.53 & 0.41 & 19.12 & 80.47 & 8.92 \\
\hline
\end{tabular}

[1] Instituto Nacional de Tecnología Agropecuaria (INTA). (2009). Cobertura del suelo de la República Argentina. Año 2006-2007 (LCCS-FAO).

[2] Vallejos, M., J. N. Volante, M. J. Mosciaro, L. M. Vale, M. L. Bustamante, and J. M. Paruelo. 2015. Transformation dynamics of the natural cover in the Dry Chaco ecoregion: A plot level geo-database from 1976 to 2012. Journal of Arid Environments. 123:3-11. https://doi.org/10.1016/j.jaridenv.2014.11.009.

[3] Unidad de Manejo del Sistema de Evaluación Forestal (UMSEF)-SADyDS. 2016. Monitoreo de la Superficie de Bosque Nativo de la República Argentina Período 2006-2016. Regiones Forestales Parque Chaqueño, Selva Misionera y Selva Tucumano Boliviana. Buenos Aires, Argentina.

[4] UNEP-WCMC y UICN. 2019. Protected Planet: The World Database on Protected Areas (WDPA, agosto 2019, Cambridge, RU: UNEP-WCM y UICN). URL: https://www.protectedplanet.net/.

100 (siendo 100 el valor máximo posible para cada dimensión) (e.g., en el caso de "extensión geográfica", la categoría "la totalidad del área geográfica" obtiene un puntaje de 100 para esa dimensión). El valor resultante de incidencia de cada presión sobre cada ecorregión es producto de la multiplicación de los valores asignados a sus cuatro dimensiones, dividido en 1000000 para que varíe entre 0 y 100 (Anexo 2). Cabe destacar que buscamos identificar las presiones que en la actualidad se ejercen en cada ecorregión. Esto quiere decir que algunas presiones que en el pasado fueron muy relevantes y configuraron la ecorregión pueden quedar relegadas en la consideración debido a que hoy se encuentran relativamente estabilizadas o decrecientes. La expansión agrícola, por ejemplo, fue muy importante para la ecorregión Selva de las Yungas, al afectar casi en su totalidad el pedemonte de la región; sin embargo, los procesos actuales de expansión agrícola son pocos y localizados en los escasos remanentes de selva de pedemonte, lo que lleva al proceso a tener un bajo valor en extensión geográfica, y por ello queda relegada en comparación con otras presiones.

Por último, representamos en un mapa las tres presiones más importantes (i.e., las tres presiones con valores más altos), y el porcentaje de la sumatoria de las restantes presiones (representado como "otras", el cual engloba el resto de las presiones) para cada una de las ecorregiones terrestres de la Argentina. El porcentaje que agrupa a las otras presiones brinda un panorama de la preponderancia de las tres primeras presiones según el criterio experto. Es decir, cuanto más elevado el porcentaje de la categoría "otras", menos preponderancia de las primeras tres presiones en una determinada ecorregión. Por el contrario, un porcentaje muy bajo de la categoría "otras" indica una fuerte preponderancia de las tres primeras presiones. 
Finalmente, es importante remarcar que, dada la naturaleza semicuantitativa del método y la subjetividad de cada equipo de trabajo en la asignación de los puntajes para cada presión, en especial los valores de aquellas presiones de menor incidencia (i.e., excepto las tres principales), deben interpretarse con cautela, ya que son particularmente sensibles a la asignación de valores sobre cada una de sus dimensiones. Es por todo esto que en este trabajo la discusión se centra sobre las tres presiones principales de cada ecorregión.

Agrupamiento de ecorregiones en función de la incidencia de las presiones asociadas al uso de la tierra

Realizamos un análisis de agrupamiento para evaluar la similitud de las distintas ecorregiones en función de los puntajes de las presiones asociadas al uso de la tierra. La técnica de agrupamiento (clustering) es un método de minería de datos que permite identificar patrones o grupos de objetos similares dentro de una base de datos de interés, de modo que la variación dentro de cada grupo (i.e., la suma de cuadrados intragrupo) se minimice (Kassambara 2017). En particular, usamos la técnica de agrupamiento jerárquico aglomerativo, la cual no requiere de la identificación a priori del número de grupos, y agrupa de forma sucesiva los objetos (en este caso las ecorregiones) en distintos grupos de acuerdo a su similitud (en este caso en el valor de cada una de las presiones). Los resultados se representan típicamente mediante un dendrograma. En un primer término, estandarizamos los datos y calculamos la similitud entre las distintas ecorregiones a través de la distancia euclideana. A partir de la matriz de distancia, utilizamos el método de agrupamiento de enlace promedio (average linkage) para realizar el dendrograma. Este método define la distancia entre dos grupos comola distancia promedio entre los elementos del grupo 1 y los elementos del grupo 2 . Una vez obtenido el dendrograma, para corroborar que las distancias representadas en el mismo reflejen las distancias originales de manera confiable, calculamos la distancia cofenética (i.e., la distancia entre dos objetos o grupos dentro del agrupamiento), y la correlacionamos con la distancia de la matriz original. En general, valores de correlación mayores a 0.75 se consideran adecuados (Kassambara 2017). Estimamos a posteriori el número óptimo de grupos utilizando el método Elbow, que calcula la suma de cuadrados intra-grupo en función del número de grupos, la cual idealmente debe ser la menor posible (Kassambara and Mundt 2020). Luego calculamos la bondad del agrupamiento con el coeficiente Silhouette, que mide el grado de pertenencia de una observación a su grupo asignado, comparando la disimilitud de cada observación i con todas las observaciones dentro del grupo al que pertenece, y de los otros grupos. El coeficiente Silhouette varía entre -1 y 1 , con valores cercanos a 1 indicando una alta pertenencia de la observación a su grupo, valores cercanos a 0 indicando la posibilidad de que la observación se encuentra entre dos grupos o que un grupo consta de un solo objeto, y valores cercanos a -1 indicando que la observación está en el grupo incorrecto (Kassambara 2017). Realizamos todos los análisis en el software $R$ versión 3.6.2. ( $R$ team 2018), utilizando el paquete factoextra (Kassambara and Mundt 2020). Para visualizar las presiones de mayor incidencia dentro de cada grupo identificado, calculamos el valor promedio de cada presión en cada grupo, y realizamos un gráfico de barras comparando los valores promedio de todas las presiones en todos los grupos.

\section{Resultados}

Los valores de superficie transformada, natural/semi-natural y protegida en cada una de las ecorregiones según las fuentes de datos a nivel nacional se reportan en la Tabla 1 . Se evidencia gran variabilidad entre ecorregiones. Por ejemplo, en cuanto a la superficie transformada, Puna y Pampa son los casos más contrastantes, con valores que oscilaron entre $0.09 \%$ y $78 \%$, respectivamente. Por otra parte, los valores de superficie protegida oscilaron entre $1.3 \%$ (Campos y Malezales, Chaco Húmedo) y 41.9\% (Bosques Patagónicos) (Tabla 1). En el Anexo 1 se presentan los documentos de síntesis y actualización de las presiones para la conservación asociadas al uso de la tierra en las ecorregiones terrestres de la Argentina, y los valores de superficie natural/semi-natural y transformada en cada una de las ecorregiones según las fuentes de datos a nivel global (Tabla A1).

\section{Identificación y jerarquización de presiones asociadas al uso de la tierra en las ecorregiones terrestres de la Argentina}

Las presiones Ganadería Comercial y Agricultura fueron identificadas como las de mayor incidencia relativa entre las ecorregiones terrestres de la Argentina. Ambas presiones alcanzaron los valores más elevados considerando la sumatoria de los 
valores obtenidos en todas las ecorregiones (Figura 1). Además, se encontraron dentro de las presiones principales en ocho y siete ecorregiones, respectivamente (Figuras 1 y 2). Por su parte, las presiones asociadas al uso de la tierra con menor incidencia regional relativa, tomando como criterio aquellas presiones cuya sumatoria es menor

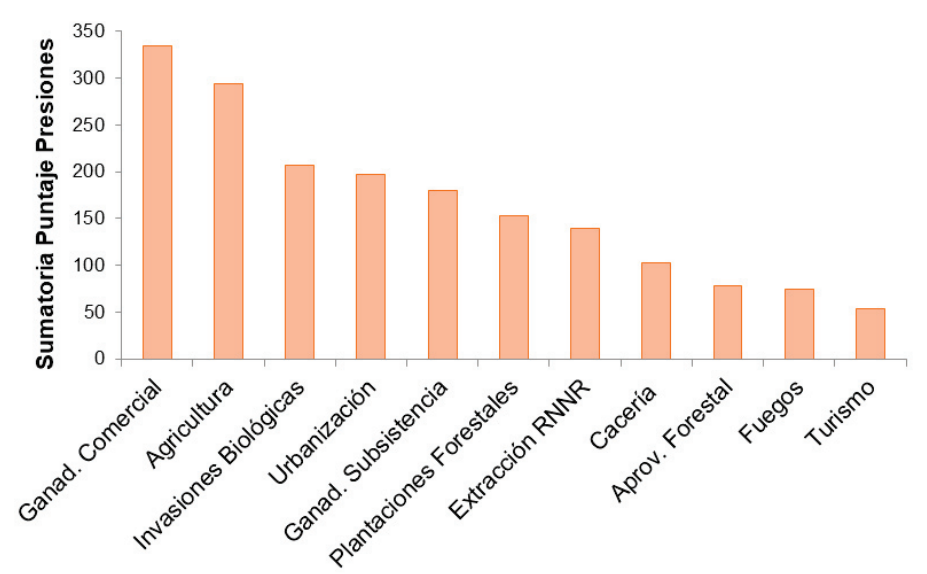

Figura 1. Incidencia de las presiones en base a la sumatoria del puntaje de cada presión (eje $Y$ ) en todas las ecorregiones terrestres de la Argentina. Ganad. Comercial: Ganadería Comercial; Urbanización: Urbanización e Infraestructura; Ganad. Subsistencia: Ganadería de Subsistencia; Extracción RNNR: Extracción de Recursos Naturales No Renovables; Aprov. Forestal BN: Aprovechamiento Forestal de Bosques Nativos; Fuegos: Fuegos de Origen Antrópico. Por su incidencia regional, la Ganadería Comercial y la Agricultura constituyen las principales presiones sobre la conservación asociadas al uso de la tierra en la Argentina.

Figure 1. Incidence of the threats based on the sum of each threat (axis y) in all the terrestrial ecoregions of Argentina. Ganad. Comercial: Commercial Livestock Production; Agricultura: Agriculture; Invasiones Biológicas: Biological Invasions; Urbanización: Urbanization and Infrastructure; Ganad. Subsistencia: Subsistence Livestock Ranching; Plantaciones Forestales: Forestry Plantations; Extracción RNNR: Extraction of Non-Renewable Resources; Cacería: Hunting; Aprov. Forestal BN: Native Forest Harvesting; Fuegos: Anthropic Fires: Turismo: Tourism. Based on their regional incidence, Commercial Livestock Production, and Agriculture are the main threats on conservation associated to land-use in Argentina.

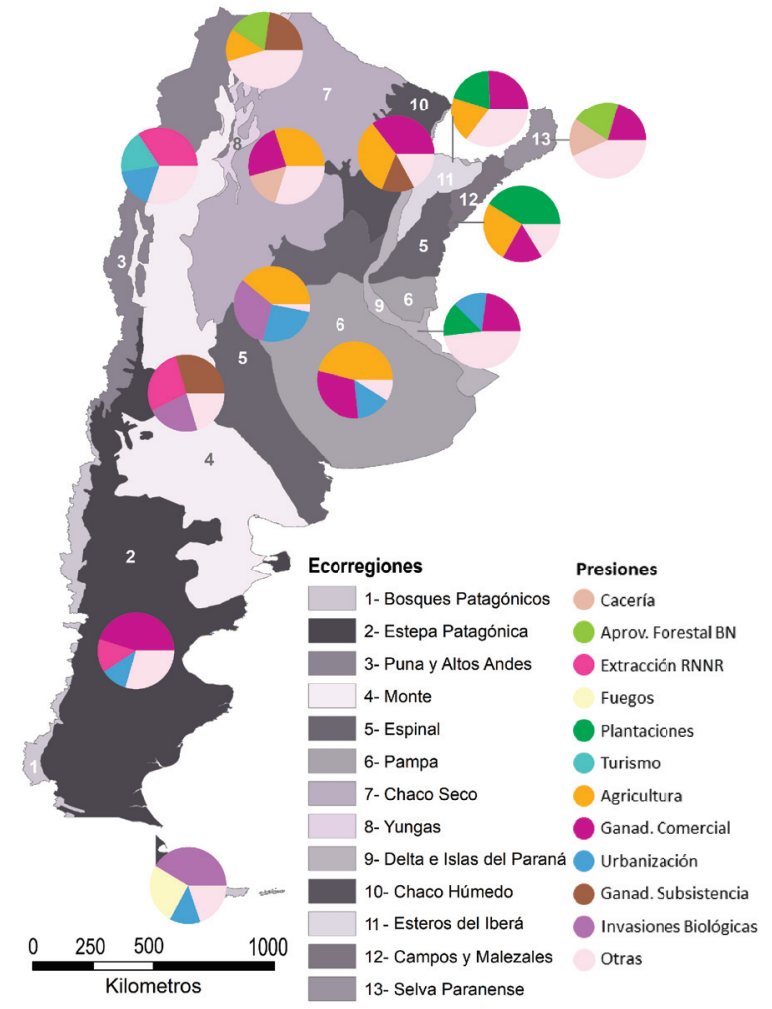

Figura 2. Representación de las tres presiones principales y su incidencia relativa, y de la sumatoria de las incidencias relativas de las presiones restantes ("Otras", representadas en rosa pálido) en cada una de las ecorregiones terrestres de la Argentina. Ganad. Comercial: Ganadería Comercial; Urbanización: Urbanización e Infraestructura; Ganad. Subsistencia: Ganadería de Subsistencia; Extracción RNNR: Extracción de Recursos Naturales No Renovables; Aprov. Forestal BN: Aprovechamiento Forestal de Bosques Nativos; Fuegos: Fuegos de Origen Antrópico. Proyección Geográfica: proyección cónica equivalente de Albers para Sudamérica.

Figure 2. Representation of the three main threats and their relative incidence, and of the sum of the relative incidences of the other threats ("Otras", in pale pink), in each of the terrestrial ecoregions of Argentina. Ganad. Comercial: Commercial Livestock Production; Agricultura: Agriculture; Invasiones Biológicas: Biological Invasions; Urbanización: Urbanization and Infrastructure; Ganad. Subsistencia: Subsistence Livestock Ranching; Plantaciones Forestales: Forestry Plantations; Extracción RNNR: Extraction of Nonrenewable Resources; Cacería: Hunting; Aprov. Forestal BN: Native Forest Harvesting; Fuegos: Anthropic Fires: Turismo: Tourism. Geographic Projection: South America Albers Equal Area Conic. 
a 100, fueron Aprovechamiento Forestal de Bosques Nativos, Fuegos por Actividades Antrópicas y Turismo (Figura 1). A pesar de estas diferencias, las 11 presiones identificadas se encontraron dentro de las tres principales en al menos una de las 13 ecorregiones analizadas (Figuras 1 y 2; Anexo 2).

\section{Análisis de similitudes entre ecorregiones en función de la incidencia de las presiones asociadas al uso de la tierra}

El agrupamiento jerárquico obtenido en función de la incidencia de las distintas presiones sobre las ecorregiones, presentó un número óptimo de grupos (i.e., que minimiza la suma de cuadrados intra-grupo en función del número de grupos) de cuatro (Figura 3). El valor del coeficiente de correlación cofenética fue 0.86, y el valor de Silhouette (S) fue 0.23, lo que indica un agrupamiento aceptable, si bien este varió entre los distintos grupos. Los cuatro grupos quedaron conformados del siguiente modo: 1 ) Monte y Puna ( $S=0.37)$, con altos valores de Ganadería de Subsistencia y Extracción de Recursos Naturales No Renovables; 2) Bosques Patagónicos ( $S=0)$, con altos valores de Invasiones Biológicas y Fuegos; 3) Selva Paranaense y Yungas ( $\mathrm{S}=0.17$ ), con altos valores de Aprovechamiento Forestal de Bosques Nativos, y 4) Espinal, Delta e Islas del Paraná, Esteros del Iberá, Campos y Malezales, Estepa Patagónica, Chaco Seco, Chaco Húmedo y Pampa $(S=0.23)$, con altos valores de Ganadería Comercial y Agricultura (Figuras 3 y 4 ).

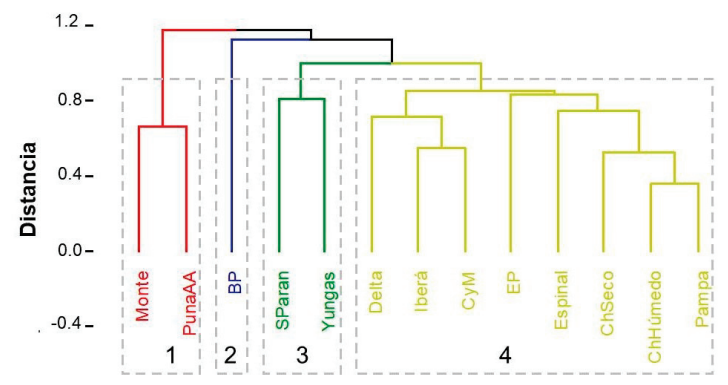

Figura 3. Dendrograma resultante del agrupamiento jerárquico de las ecorregiones en función de la incidencia de las distintas presiones. Grupo 1: Monte, Puna y Altos Andes (PunaAA); Grupo 2: Bosques Patagónicos (BP); Grupo 3: Selva Paranaense (SParan) y Selva de las Yungas (Yungas); Grupo 4: Campos y Malezales (CyM), Chaco Seco (ChSeco), Chaco Húmedo (ChHúmedo), Delta e Islas del Paraná (Delta), Esteros del Iberá (Iberá), Espinal, Estepa Patagónica (EP) y Pampa. Figure 3. Dendrogram of the hierarchical clustering of ecoregions based on the incidence of the different threats. Group 1: Monte, Puna and Altos Andes (PunaAA); Group 2: Bosques Patagónicos (BP); Group 3: Selva Paranaense (SParan) and Selva de las Yungas (Yungas); Group 4: Campos y Malezales (CyM), Chaco Seco (ChSeco), Chaco Húmedo (ChHúmedo), Delta e Islas del Paraná (Delta), Esteros del Iberá (Iberá), Espinal, Estepa Patagónica (EP) and Pampa.

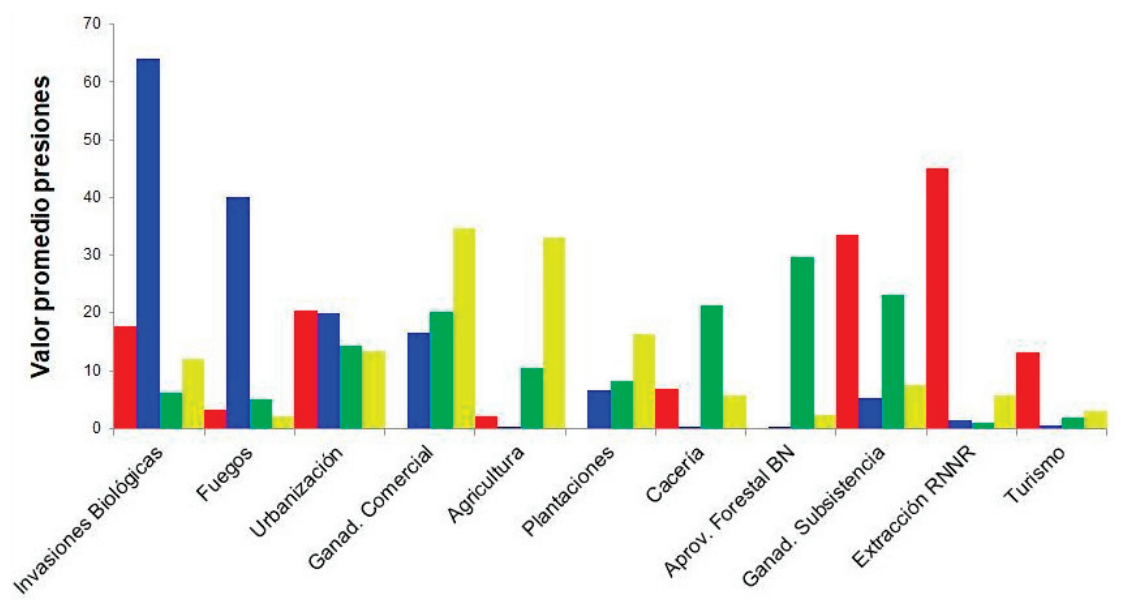

Figura 4. Valores promedio de las presiones dentro de cada grupo derivado del análisis de agrupamiento. Grupo 1 (Monte, Puna y Altos Andes): rojo; Grupo 2 (Boques Patagónicos): azul; Grupo 3 (Selva Paranaense y Selva de las Yungas): verde; Grupo 4 (Campos y Malezales, Chaco Húmedo, Chaco Seco, Delta e Islas del Paraná, Estepa Patagónica Espinal, Esteros del Iberá, Pampa): amarillo. Figure 4. Mean values of the threats within each group derived from the cluster analysis. Group 1 (Monte, Puna and Altos Andes): red; Group 2 (Boques Patagónicos): blue; Group 3 (Selva Paranaense y Selva de las Yungas: green; Group 4 (Campos y Malezales, Chaco Húmedo, Chaco Seco, Delta e Islas del Paraná, Estepa Patagónica, Espinal, Esteros del Iberá, Pampa): yellow. 


\section{DISCUSIÓN}

Las presiones asociadas al uso de la tierra con mayor incidencia relativa en las ecorregiones terrestres de la Argentina, en base a su extensión geográfica, probabilidad de ocurrencia, severidad e irreversibilidad, fueron la Ganadería Comercial y la Agricultura (Figuras 1 y 2). Las mismas se encontraron dentro de las principales presiones en ocho y siete de las 13 ecorregiones terrestres de la Argentina, respectivamente. En particular, cinco presentaron una influencia conjunta de la Ganadería Comercial y la Agricultura como principales presiones asociadas al uso de la tierra (Campos y Malezales, Esteros del Iberá, Chaco Húmedo, Chaco Seco y Pampa). De hecho, el agrupamiento realizado en este trabajo separó a las ecorregiones terrestres argentinas en grupos principalmente en función de la incidencia de estas dos presiones (i.e., ecorregiones agrícolas [grupo 4], y noagrícolas [grupos 1, 2 y 3]). Estos resultados son concordantes con la situación mundial actual, en la que ambos usos de la tierra se reportan como la principal presión sobre la conservación de los ecosistemas terrestres (Díaz 2019; IPBES 2019).

En la Argentina, la expansión y la intensificación de la agricultura y de la ganadería comercial se ven favorecidas por características ambientales y socioeconómicas locales propias de cada ecorregión (e.g., el tipo de suelo, la topografía, las precipitaciones o la disponibilidad de una mayor infraestructura [Grau et al. 2005; Piquer-Rodríguez et al. 2018]), factores nacionales (e.g., los acuerdos comerciales y las aperturas de nuevos mercados [Gasparri et al. 2009]), y factores globales (e.g., el constante aumento en la demanda de alimentos y el crecimiento de la población [Grau et al. 2005; Fróna et al. 2019]). A su vez, la agricultura y la ganadería comercial suelen ocurrir de manera acoplada, lo cual explica la incidencia de ambas presiones en simultáneo en muchas de las ecorregiones terrestres de la Argentina. Esto quiere decir que ambas actividades están vinculadas entre sí por flujos de capital, insumos y productos, comparten actores y tomadores de decisiones que operan en ambos tipos de uso de la tierra, y habitualmente avanzan en una secuencia temporal y espacial en la forma de ocupar el territorio (i.e., nuevas áreas de vegetación natural se reemplazan por ganadería, que luego se reemplaza por agricultura) (Gasparri et al. 2013; Gasparri and Le Polain de Waroux 2015).
En ecorregiones con menor aptitud agrícola se observa una mayor incidencia de otras presiones de menor relevancia a nivel nacional (e.g., Aprovechamiento Forestal, Ganadería de Subsistencia) o, inclusive, la ocurrencia en simultáneo de presiones que hoy en día se encuentran en expansión, junto a otras asociadas a usos de la tierra más tradicionales (e.g., Agricultura, Ganadería Comercial y Cacería en el Chaco Seco). Estas presiones más puntuales operan más específicamente sobre ecorregiones de menor aptitud agrícola, y en muchos casos de alto valor de conservación (e.g., selvas subtropicales húmedas), y suelen vincularse a usos de la tierra más tradicionales, de agentes forzantes locales y de poca incidencia económica a nivel nacional (e.g., Cacería, Ganadería de Subsistencia). Por ello, es posible que requieran del diseño de políticas y estrategias de manejo a una escala más local, con una fuerte consideración de los intereses de las poblaciones locales (cuyas economías y bienestar dependen de usos de la tierra tradicionales).

A partir del análisis de agrupamiento identificamos cuatro grupos de ecorregiones que se discuten a continuación (Figura 3). Es importante destacar que los agrupamientos de ecorregiones en función de presiones comunes indican similitudes entre las mismas, pero esto no significa que no estén operando presiones particulares de cada ecorregión.

\section{Monte, Puna y Altos Andes}

Las tres ecorregiones áridas del oeste de la Argentina - el Monte, la Puna y los Altos Andes - se agrupan en un módulo en función de una relativamente alta incidencia de la extracción de Recursos Naturales No Renovables y de Ganadería de Subsistencia (Figuras 2 y 4; Anexo 2). En el caso del Monte, la principal presión asociada al uso de la tierra identificada actualmente es la ganadería de subsistencia. Esta constituye un uso de la tierra crónico (desde el siglo XIX) extendido en toda la ecorregión, que contribuye de forma ocasional a procesos de desertificación con diversas consecuencias ambientales (Anexo 1). En el caso de la Puna y los Altos Andes, si bien la ganadería de subsistencia es una de las principales actividades económicas (y según nuestro análisis se encuentra como cuarta presión), en la actualidad está en un proceso de disminución debido a los cambios socioeconómicos recientes vinculados a otros usos de la tierra (Grau et al. 2018; Izquierdo et al. 2018). Tanto en el Monte como en la 
Puna y los Altos Andes, la extracción minera (metales preciosos y petróleo en el norte y sur del Monte, respectivamente, y extracción de minerales en la Puna y los Altos Andes) está ampliamente distribuida y en expansión en las últimas décadas, vinculada a agentes forzantes distantes (Izquierdo et al. 2015, 2018). En estas ecorregiones, la extracción minera tiene diversas consecuencias socioambientales, y a su vez, se asocia a otros procesos, como la urbanización y la expansión de la infraestructura, y en algunos sectores de la Puna, incluso con el turismo. Este último puede presentar implicancias socioeconómicas y ambientales favorables, pero requiere de la implementación de políticas de planificación y manejo, en especial en relación con los cambios sociales y culturales que implica (Izquierdo et al. 2018). En la zona del Monte, la implementación de la Ley de Presupuestos Mínimos para la Conservación de Bosques Nativos (Ley N²6.331) permitió promover un uso sustentable de los bosques del centro-norte de este bioma, en concordancia con políticas provinciales de conservación, como en Mendoza, Catamarca y La Rioja (Villagra and Álvarez 2019). Cabe destacar que el Monte, la Puna y los Altos Andes presentan un alto valor de conservación, a pesar de no presentar una alta riqueza de especies, debido a la presencia de especies endémicas y amenazadas (Cajal and Bonaventura 1998; Ojeda 2002). Por último, es importante aclarar que el haber analizado la Puna y los Altos Andes, y el Monte de Llanuras y Mesetas y de Sierras y Bolsones en un mismo diagnóstico constituye una limitación que posiblemente enmascare presiones propias de cada ecorregión (Burkart et al. 1999).

\section{Bosques Patagónicos}

Los Bosques Patagónicos no se agruparon con otra ecorregión terrestre de la Argentina, por la singularidad de sus presiones detectadas, dentro de las que Invasiones Biológicas y Fuegos de Origen Antrópico adquirieron los valores más elevados (Figuras 2 y 4; Anexo 2). Las invasiones biológicas constituyen una problemática importante en esta ecorregión, e involucran especies vegetales y animales de sistemas terrestres y acuáticos (ver Anexo 1). A su vez, los fuegos de origen antrópico son quizás el disturbio más importante (Veblen et al. 2003), lo cual se refleja en nuestra valoración de presiones. Sin embargo, en comparación, los Bosques Patagónicos presentan una muy baja superficie transformada, y la mayor superficie de áreas protegidas en relación a las otras ecorregiones terrestres de la Argentina (Tabla 1). Además, y a pesar de representar una baja proporción del área, la Urbanización y Expansión dela Infraestructura fue identificada como la tercera presión sobre esta ecorregión, principalmente por presentar escasa regulación y planificación, $\mathrm{y}$ por vincularse a diversos problemas indirectos asociados (e.g., contaminación del agua, acumulación de basura, extracción de áridos, desmonte para pistas de esquí y otras actividades turísticas, caza furtiva, impacto sobre cuencas) (Mateucci 2012).

\section{Selva Paranaense y Selva de las Yungas}

Las selvas subtropicales de la Argentina (Selva Paranaense y Yungas) se encontraron en un mismo grupo, ambas caracterizadas por altos valores de Aprovechamiento Forestal, Cacería, Uso para Ganadería de Subsistencia y Ganadería Comercial (Figuras 2 y 4; Anexo 2). Además, las selvas subtropicales de la Argentina albergan la mayor parte de la biodiversidad del país, por lo cual poseen un alto valor de conservación (Anexo 1). Una diferencia entre ambas ecorregiones es el marcado gradiente altitudinal y latitudinal en la ecorregión de Yungas, que abarca diferentes límites políticos provinciales con una desigual incidencia de las presiones asociadas al uso de la tierra. Por ejemplo, mientras que en las Yungas del norte la explotación forestal continúa siendo una presión importante, en las Yungas del sur presenta más relevancia la expansión de especies exóticas (Anexo 1) (Izquierdo and Grau 2009). A su vez, en la Selva Pedemontana de Yungas, las presiones asociadas al uso de la tierra más relevantes son la agricultura y la urbanización, pero en sectores de Selva Montana y Bosque Montano las presiones están más vinculadas a actividades tradicionales, como la ganadería de subsistencia, y la cacería de subsistencia o como respuesta a la depredación de ganado (Blundo et al. 2012) (Anexo 1). El aprovechamiento forestal de bosques nativos es una presión transversal a todos los pisos altitudinales en las Yungas, lo cual la ubicó como la segunda presión principal asociada al uso de la tierra según nuestro análisis (Figura 2) (Politi et al. 2012). Por su parte, en la Selva Paranaense, el aprovechamiento forestal también es importante, y su presión (al igual que las presiones ejercidas por la cacería y la ganadería), se distribuye de manera más homogénea a lo largo del 
territorio. Sin embargo, es necesario resaltar que esta ecorregión se encuentra en el límite de tres países, con contextos socioeconómicos particulares que influyen sobre las políticas de conservación.

\section{Campos y Malezales, Chaco Húmedo,} Chaco Seco, Delta e Islas del Paraná, Estepa Patagónica, Esteros del Iberá y Pampa

Las ecorregiones agropecuarias del país conformaron un mismo grupo en el análisis de agrupamiento, sobre todo por sus altos valores de Ganadería Comercial o Agricultura (en seis de estas ecorregiones ambas presiones se encontraron dentro de las tres principales) (Figuras 2 y 4; Anexo 2). En consecuencia, estas ecorregiones se caracterizan también por altos valores de superficie transformada, en general asociados a bajos valores de superficie protegida (Tabla 1). La Estepa Patagónica constituye una excepción, con bajos niveles de superficie transformada debido a que la ganadería comercial se practica de forma extensiva y con una dominancia de ganado ovino (Anexo 1).

A pesar de la dominancia de la agricultura o ganadería comercial dentro de las presiones que caracterizan a este grupo, otras presiones adquirieron relevancia en subgrupos de ecorregiones, lo que las separa en el análisis de agrupamiento (Figura 3). Por ejemplo, el subgrupo integrado por Campos y Malezales, Delta e Islas del Paraná y Esteros del Iberá se distingue por valores altos de Plantaciones Forestales (Anexo 2). La expansión de las plantaciones en estas tres ecorregiones es, en general, reciente (posterior a 1990), y en Campos y Malezales y en Esteros del Iberá involucra el reemplazo de bosques nativos y pastizales naturales por plantaciones de especies exóticas leñosas para el uso maderero, económicamente más rentables y de rápido crecimiento (Matteucci et al. 2012; Neiff and Poi de Neiff 2005). En cambio, en el bajo Delta (Delta e Islas del Paraná) las plantaciones forestales de salicáceas se establecen bajo diques (Sica et al. 2016). Por otra parte, esta ecorregión se destaca por su cercanía a importantes centros urbanos y por tratarse de un ecosistema de humedales, con lo cual atraviesa grandes procesos de transformación asociados a diversos usos de la tierra, que adquirieron valores simultáneamente altos en nuestra evaluación (e.g., urbanización, distintos tipos de plantaciones forestales, ganadería comercial, invasiones biológicas) (Anexo 1). En cambio, en los Esteros del
Iberá, el reciente establecimiento de ONG ambientalistas impulsaron actividades de ecoturismo y la creación y el fortalecimiento de las áreas protegidas a través del gobierno provincial y nacional, lo que contribuye a la conservación de la ecorregión (Anexo 1).

El subgrupo conformado por el Chaco Seco, el Chaco Húmedo, el Espinal, la Estepa Patagónica y la Pampa se caracterizaron por altos valores de presión de Agricultura o Ganadería Comercial (Figuras 2 y 4; Anexo 2). La Pampa y el Espinal tienen una larga historia de expansión agrícola anterior al de Chaco Seco y Chaco Húmedo. La expansión de la ganadería ocurrió desde el siglo XVI asociada al inicio de la colonización europea, seguida por el reemplazo por cultivos de girasol, maíz y trigo a principios del siglo XX, y por soja a partir de 1990. Esto último desplazó la ganadería a sectores marginales del Chaco y del Espinal (Viglizzo et al. 2011). En ese sentido, la actual intensificación agrícola y ganadera en la Pampa es el proceso dominante, mientras que en el Chaco Seco y el Chaco Húmedo lo son tanto la expansión como la intensificación (Baumman et al. 2017; Piquer et al. 2018). Las ecorregiones del Chaco Seco y Húmedo son incorporadas al control estatal de manera más tardía, y la ocupación del territorio con procesos de deforestación y arbustificación de pastizales naturales ocurre recién entre finales del siglo XIX y principios del siglo XX, asociados a planes de colonización y a la expansión de la ganadería (Grau et al. 2014; Le Polain et al. 2018). Además, estas ecorregiones se caracterizan por presentar una alta incidencia de ganadería de subsistencia, asociada a las comunidades campesinas e indígenas de la región, las cuales, en general, tienen una tenencia precarizada de la tierra y muestran dificultades para insertarse en los nuevos modelos de producción (Mastrángelo and Laterra 2015). A su vez, la ganadería de subsistencia se vincula a la degradación de las tierras, y parece asociarse a la cacería por conflictos con carnívoros por depredación de ganado (Adamoli et al. 1990; Quiroga et al. 2014, 2016). En estos contextos, resulta fundamental entender las interacciones entre usos de la tierra tradicionales y modernos que tienen lugares en estas ecorregiones dinámicas, y las consecuencias socioambientales de dichas interacciones.

En el Espinal, la Urbanización e Infraestructura fue identificada como una presión relevante, en general asociada a la expansión de la frontera agropecuaria y a la 
cercanía a grandes centros urbanos (Gasparri et al. 2015; Piquer-Rodríguez et al. 2018). En el caso de esta ecorregión, consideramos importante resaltar que incluye sectores con situaciones contrastantes, ya reflejadas en la vegetación natural, con tres tipos de bosques o sabanas (distrito del Nandubay, distrito del Algarrobo y distrito del Caldenal) (Matteucci et al. 2012). En nuestra opinión, las zonas del Caldenal y del Nandubay empiezan a tener presiones y usos distintivos que requieren de un análisis más específico para cada uno de los sectores.

\section{Consideraciones y alcance del diagnóstico}

Nuestros resultados brindan un diagnóstico general de las principales presiones asociadas al uso de la tierra que operan sobre las ecorregiones terrestres de la Argentina. En ese sentido, es importante destacar que se trata de una aproximación semicuantitativa, basada en criterio experto, a través de un método que evalúa ciertas dimensiones de esas presiones (i.e., probabilidad de ocurrencia, extensión, severidad, reversibilidad). Si bien este tipo de evaluaciones más sistemáticas tienen un componente subjetivo, su uso proporciona un mecanismo para fundamentar la opinión de expertos/as, que permite transparentar criterios y orientar discusiones y cuestionamientos enriquecedores (algunos de los cuales se dieron internamente entre los/las autores/as de este trabajo, e inclusive durante las rondas de evaluación de esta revista). En última instancia, estas discusiones contribuyen a identificar vacíos de información y aspectos controversiales que requieran de análisis cuantitativos más detallados. Discutimos algunos de estos puntos a continuación.

1) El método seleccionado de jerarquización de presiones asigna igual ponderación a las cuatro dimensiones de las mismas (i.e., probabilidad de ocurrencia, severidad, extensión e irreversibilidad), asumiendo que cada dimensión influye de igual manera sobre la magnitud de la presión. A su vez, la relevancia de cada presión cobra sentido en relación a su posición relativa con respecto a las otras presiones analizadas dentro de una ecorregión determinada, pero no necesariamente entre ecorregiones. Por otra parte, cabe señalar que hay ecorregiones donde aún las principales presiones detectadas son bajas. La Urbanización, por ejemplo, representa menos del $2 \%$ del área en todas las ecorregiones, de modo que aunque es identificada como una de las tres presiones principales, su efecto al menos en términos de superficie afectada es bajo.

2) Si bien los diagnósticos en cada ecorregión se hicieron de manera coordinada y siguiendo un método sistemático para tomar las decisiones, pueden existir algunas diferencias de criterio entre regiones. En ese sentido, es importante destacar que la asignación de valores a las presiones y, por lo tanto, el ranking de presiones es, en última instancia, el resultado primario del criterio de los/las expertos/as de cada ecorregión, los que después se sometieron a una discusión plenaria presencial en busca de la mayor coherencia y consenso posible entre regiones, y a numerosas discusiones posteriores durante la redacción. De esta manera, los puntajes asignados se deben interpretar y justificar junto con las descripciones detalladas de cada ecorregión (Anexo 1). La subjetividad propia de este tipo de enfoques metodológicos se podría controlar, por ejemplo, a través de un diagnóstico colectivo en todas las ecorregiones como alternativa a la evaluación independiente de cada ecorregión por los respectivos grupos de trabajo, o a través de validaciones con grupos expertos externos.

3) Las presiones no actúan de manera aislada, sino que son dinámicas en el tiempo y el espacio, y existen interacciones positivas y negativas entre ellas que no fueron consideradas de manera explícita en esta evaluación. Por ejemplo, la urbanización, la agricultura intensiva, la minería o el crecimiento del turismo pueden significar presiones localmente intensas e importantes para algunas ecorregiones, pero a su vez pueden derivar en la disminución de otras presiones, como la agricultura en tierras marginales, la ganadería de subsistencia o la cacería, en esa misma ecorregión u otra. La acción conjunta de la presión y sus interacciones requieren trabajos específicos para evaluar las consecuencias sobre la conservación a escala regional y en sistemas tele-acoplados, lo cual excede el alcance de este trabajo y de los métodos utilizados.

4) En ecorregiones heterogéneas (e.g., Espinal, Monte o Selva de Yungas), este tipo de diagnósticos sería más preciso si se realizara en las distintas subregiones (e.g., distritos en el Espinal, pisos altitudinales en las Yungas), debido a que la influencia de distintas presiones sobre las mismas puede ser localmente muy distinta y verse enmascarada por un diagnóstico a escala 
ecorregional. Alternativamente, se podría considerar el reemplazo de la zonificación por ecorregiones (con una base fundada puramente en los ecosistemas naturales) por un esquema que considere de manera explícita las interacciones entre naturaleza y sociedad (e.g., Ellis and Ramankutty 2008).

\section{CONCLUSIONES}

Este trabajo contribuye a actualizar diagnósticos previos de las presiones que operan sobre las ecorregiones terrestres de la Argentina (e.g., Brown et al. 2006). El método utilizado para identificar las principales presiones en cada ecorregión constituye una aproximación superadora, al transparentar y poner de manera explícita los criterios empleados (i.e., puntajes) para la identificación de las presiones relevantes. Los resultados obtenidos pueden ser tomados como un punto de partida para estudios específicos que permitan evaluar de manera más detallada los vínculos entre usos de la tierra y conservación para diferentes problemáticas, ecorregiones, o subregiones dentro de estas.

La existencia de presiones comunes en ecorregiones distintas, y en algunos casos distantes en la geografía, pone de manifiesto la utilidad de políticas integradas de gestión de los recursos naturales que excedan las divisiones político-administrativas, y en algunos casos de ecorregiones. E1 agrupamiento más evidente entre las ecorregiones terrestres de la Argentina fue el de ecorregiones agrícolas y no-agrícolas, en general resultado de diferencias en las características biofísicas que condicionan el tipo de actividades humanas que se pueden realizar. Si bien a partir de este aporte se visibilizan conjuntos de ecorregiones con presiones asociadas al uso de la tierra comunes, los resultados obtenidos ponen también en evidencia la necesidad de realizar evaluaciones más detalladas de las diferentes problemáticas, especialmente en ecorregiones heterogéneas.

Agradecimientos. Agradecemos a la fundación Rufford por el financiamiento para la realización del taller presencial relacionado al presente trabajo; y a la Secretaría de Posgrado de la Facultad de Cs. Naturales, y la Facultad de Cs. Naturales (UNT, Tucumán) por brindar el espacio físico para la realización del taller. Agradecemos también al editor asociado de Ecología Austral y los dos revisores por sus valiosas contribuciones que mejoraron sustancialmente la calidad del manuscrito.

\section{REFERENCIAS}

Adamoli, J., E. Sennhauser, J. M. Acero, and A. Rescia. 1990. Stress and disturbance: vegetation dynamics in the dry Chaco region of Argentina. Journal of Biogeography 17(4/5):491-500. https://doi.org/ 10.2307/2845381.

Aguiar, S., M. E. Mastrangelo, M. A. G. Collazo, G. H. C. Sans, C. E. Mosso, L. Ciuffoli, M. Schmidt, M. Vallejos, L. Langbehn, M. Brassiolo, D. Cáceres, G. Merlinsky, J. M. Paruelo, L. Seghezzo, L. Staiano, M. Texeira, J. N. Volante, and S. R. Verón. 2018. ¿Cuál es la situación de la Ley de Bosques en la Región Chaqueña a diez años de su sanción? Revisar su pasado para discutir su futuro. Ecología Austral 28(2):400-417. https://doi.org/10.25260/EA.18.28.2.0.677.

Aide, T. M., M. L. Clark, H. R. Grau, D. LópezロCarr, M. A. Levy, D. Redo, M. BonillaaMoheno, G. Riner, M. J. AndradeロNúñez, and M. Muñiz. 2013. Deforestation and Reforestation of Latin America and the Caribbean (20012010). Biotropica 45(2):262-271. https:/ / doi.org/10.1111/j.1744-7429.2012.00908.x.

Baldi, G., S. Schauman, M. Texeira, S. Marinaro, O. A. Martin, P. Gandini, and E. G. Jobbágy. 2019. Nature representation in South American protected areas: country contrasts and conservation priorities. PeerJ 7:e7155. https://doi.org/ $10.7717 /$ peerj.7155.

Ballari S. A., C. B. Anderson, and A. E. Valenzuela. 2016. Understanding trends in biological invasions by introduced mammals in southern South America: a review of research and management. Mammal Review 46(3):229-240. https: //doi.org/10.1111/mam.12065.

Baumann M., I. Gasparri, M. PiquernRodríguez, G. Gavier Pizarro, G. Griffiths, P. Hostert, and T. Kuemmerle. 2017. Carbon emissions from agricultural expansion and intensification in the Chaco. Global Change Biology 23(5):19021916. https://doi.org/10.1111/gcb.13521.

Blundo, C., L. R. Malizia, J. G. Blake, and A. D. Brown. 2012. Tree species distribution in Andean forests: influence of regional and local factors. Journal of Tropical Ecology 28(1):83-95. https://doi.org/10.1017/S0266467411000617

Brondizio, E. S., J. Settele, S. Díaz, and H. T Ngo. 2019. Global assessment report on biodiversity and ecosystem services of the Intergovernmental Science-Policy Platform on Biodiversity and Ecosystem Services. IPBES Secretariat.

Brown, A., U. Martínez Ortiz, M. Acerbi, and J. F. Corcuera. 2006. La situación ambiental Argentina 2005. Fundación Vida Silvestre Argentina, Buenos Aires.

Burkart, R., N. O. Bárbaro, R. O. Sánchez, and D. A. Gómez. 1999. Eco-regiones de la Argentina. Presidencia de la Nación-Secretaría de Recursos Naturales y Desarrollo Sustentable-Administración de Parques Nacionales.

Cabrera, Á. L. 1976. Regiones fitogeográficas argentinas. Pp. 1-85 en W. F. Kugler (ed.). Enciclopedia Argentina de Agricultura y Jardinería. Fascículo 1. Tomo 2, 2a ed. Acme. Buenos Aires. Argentina. 
Cajal, J. L., and S. M. Bonaventura. 1998. Densidad poblacional y dinámica de los grupos familiares de guanacos y vicuñas en la Reserva de Biosfera de San Guillermo. Pp. 161-167 en J. L. Cajal, J. J. García Fernández and R. Tecchi (eds.). Bases para la conservación y manejo de la puna y cordillera frontal de la Argentina: El rol de las reservas de biosfera. UNESCO (Uruguay)-FUCEMA, Montevideo. Uruguay.

Campanello, P. I., J. von Below, N. I. Hilgert, K. Cockle, M. Villagra, D. di Francescantonio, D. S. García, M. Jaramillo, O. A. Gauto, and G. Goldstein. 2019. Is sustainable forest harvesting possible in Misiones? The need for multi-scale management, research, high-impact interventions, and funding. Ecología Austral 29(01):122-137. https://doi.org/ 10.25260/EA.19.29.1.0.756.

CBD, U. 2010. Strategic plan for biodiversity 2011-2020 and the Aichi targets. In Report of the Tenth Meeting of the Conference of the Parties to the Convention on Biological Diversity.

Costanza R., R. d’Arge, R. De Groot, S. Farber, M. Grasso, B. Hannon, K. Limburg, S. Naeem, R. V. O’Neill, J. Paruelo, R. G. Raskin, P. Sutton, and M. van den Belt. 1997. The value of the world's ecosystem services and natural capital. Nature 387(6630):253. https://doi.org/10.1038/387253a0

Daily G. C. 1997. Introduction: what are ecosystem services? Pp. 1-10 in G. C. Daily (ed.). Nature's Services: Societal Dependence on Natural Ecosystems. Island Press, Washington DC.

S. Díaz, J. Settele, E. Brondízio, H. Ngo, M. Guèze, J. Agard, A. Arneth, P. Balvanera, K. Brauman, S. Butchart, K. Chan, L. Garibaldi, K. Ichii, J. Liu, S. Subrmanian, G. Midgley, P. Miloslavich, Z. Molnár, D. Obura, A. Pfaff, S. Polasky, A. Purvis, J. Razzaque, B. Reyers, R. Chowdhury, Y. Shin, I. Visseren-Hamakers, K. Wilis, and C. Zayas. 2019. Summary for policymakers of the global assessment report on biodiversity and ecosystem services-unedited advance version. Intergovernmental Science-Policy Platform on Biodiversity and Ecosystem Services (IPBES), Bonn, Germany.

Dinerstein E., C. Vynne, E. Sala, A. R. Joshi, S. Fernando, T. E. Lovejoy, J. Mayorga, D. Olson, G. P. Asner, J. E. M. Baillie, N. D. Burgess, K. Burkart, R. F. Noss, Y. P. Zhang, A. Baccini, T. Birch, N. Hahn, L. N. Joppa, and E. Wikramanayake. 2019. A global deal for nature: Guiding principles, milestones, and targets. Science Advances 5(4):eaaw2869. https://doi.org/10.1126/sciadv.aaw2869. Dormann, C. F., and R. Strauss. 2014. A method for detecting modules in quantitative bipartite networks. Methods in Ecology and Evolution 5(1):90-98. https://doi.org/10.1111/2041-210X.12139.

Dormann, C. F., J. Fruend, and B. Gruber. 2019. Visualising Bipartite Networks and Calculating Some (Ecological) Indices. R-Package. URL: https://github.com/biometry/bipartite.

Edwards, P. J., and C. Abivardi. 1998. The value of biodiversity: where ecology and economy blend. Biological Conservation 83(3):239-246. https://doi.org/10.1016/S0006-3207(97)00141-9.

Ellis, E. C., and N. Ramankutty. 2008. Putting people in the map: anthropogenic biomes of the world. Frontiers in Ecology and the Environment 6(8):439-447. https://doi.org/10.1890/070062.

Fróna D., J. Szenderák, and M. Harangi-Rákos. 2019. The Challenge of Feeding the World. Sustainability 11(20):5816. https://doi.org/10.3390/su11205816.

Gasparri N. I., and H. R. Grau. 2009. Deforestation and fragmentation of Chaco dry forest in NW Argentina (1972-2007). Forest Ecology and Management 258(6):913-921. https:/ / doi.org/10.1016/j.foreco.2009.02.024.

Gasparri, N. I., and Y. L. P. de Waroux. 2015. The coupling of South American soybean and cattle production frontiers: new challenges for conservation policy and land change science. Conservation Letters 8(4):290-298. https://doi.org/ 10.1111/conl.12121.

Gasparri, N. I., H. R. Grau, and L. V. Sacchi. 2015. Determinants of the spatial distribution of cultivated land in the North Argentine Dry Chaco in a multi-decadal study. Journal of arid environments 123:31-39. https://doi.org/ 10.1016/j.jaridenv.2015.05.005.

Grau, H. R., N. I. Gasparri, and T. M. Aide. 2005. Agriculture expansion and deforestation in seasonally dry forests of north-west Argentina. Environmental Conservation 32(2):140-148. https://doi.org/10.1017/S0376892905002092.

Grau, H. R., N. I. Gasparri, and T. M. Aide. 2008. Balancing food production and nature conservation in the Neotropical dry forests of northern Argentina. Global Change Biology 14(5):985-997. https://doi.org/10.1111/ j.1365-2486.2008.01554.x.

Grau, H. R., R. Torres, N. I. Gasparri, P. G. Blendinger, S. Marinaro, and L. Macchi. 2015. Natural grasslands in the Chaco. A neglected ecosystem under threat by agriculture expansion and forest-oriented conservation policies. Journal of Arid Environments 123:40-46. https://doi.org/10.1016/j.jaridenv.2014.12.006.

Grau, H. R., A. Izquierdo, J. Babot, and A. Grau. 2018. La Puna Argentina: naturaleza y cultura. Serie Conservación de la Naturaleza, Fundación Miguel Lillo, Tucumán.

Hansen, A. J., R. S. DeFries, and W. Turner. 2012. Land use change and biodiversity. Pp. 277-299 in G. Gutman, A. C. Janetos, C. O. Justice, F. F. Moran, J. F. Mustard, R. R. Rindfuss, D. Skole, B. L. Turner II and M. A. Cochrane (eds.). Land change science. Springer, Dordrecht.

INTA. 2009. Informe Técnico Unificado PNECO 1643. Cobertura del suelo de la República Argentina. Año 2006-2007 (LCCS-FAO). INTA, Buenos Aires.

IPCC. 2014. Synthesis Report. Contribution of working groups I. ii and iii to the fifth assessment report of the intergovernmental panel on climate change. Pp. 138.

Izquierdo, A. E., and H. R. Grau. 2009. Agriculture adjustment, land-use transition and protected areas in Northwestern Argentina. Journal of Environmental Management 90(2):858-865. https://doi.org/10.1016/i.jenvman.2008.02.013.

Izquierdo, A. E., J. Foguet, and H. R. Grau. 2015. Mapping and spatial characterization of Argentine High Andean peatbogs. Wetlands ecology and management 23(5):963-976. http:/ /dx.doi.org/10.1007/s11273-015-9433-3.

Izquierdo, A. E., H. R. Grau, C. J. Navarro, E. Casagranda, M. C. Castilla, and A. Grau. 2018. Highlands in Transition: 
Urbanization, Pastoralism, Mining, Tourism, and Wildlife in the Argentinian Puna. Mountain Research and Development 38(4):390-401. https:// doi.org/10.1659/MRD-JOURNAL-D-17-00075.1.

Jabbour, J., and C. Flachsland. 2017.40 years of global environmental assessments: A retrospective analysis. Environmental Science and Policy 77:193-202. https://doi.org/10.1016/j.envsci.2017.05.001.

Kristensen P. 2004. The DPSIR framework. National Environmental Research Institute, Denmark, 10.

Lantschner M. V., V. Rusch and J. P. Hayes. 2013. Do exotic pine plantations favor the spread of invasive herbivorous mammals in Patagonia? Austral Ecology 38(3): 338-345. https://doi.org/10.1111/j.1442-9993.2012.02411.x.

Le Polain de Waroux Y., M. Baumann, N. I. Gasparri, G. Gavier-Pizarro, J. Godar, T. Kuemmerle, R. Müller, F. Vázquez, J. Norberto Volante, and P. Meyfroidt. 2018. Rents, actors, and the expansion of commodity frontiers in the Gran Chaco. Annals of the American Association of Geographers 108(1):204-225. https://doi.org/10.1080/ 24694452.2017.1360761

Licata, J. A., J. E. Gyenge, M. E. Fernández, T. M. Schlichter, and B. J. Bond. 2008. Increased water use by ponderosa pine plantations in northwestern Patagonia, Argentina compared with native forest vegetation. Forest Ecology and Management 255(3):753-764. https://doi.org/10.1016/j.foreco.2007.09.061.

Luque, S., G. M. Pastur, C. Echeverría, and M. J. Pacha. 2011. Overview of biodiversity loss in South America: a landscape perspective for sustainable forest management and conservation in temperate forests. Pp. 352-379 in C. Li, R. Lafortezza and J. Chen (eds.). Landscape Ecology in Forest Management and Conservation. Springer, Berlin, Heidelberg. https://doi.org/10.1016/j.foreco.2007.09.061.

Mace, G. M. 2014. Whose conservation? Science 345(6204):1558-1560. https://doi.org/10.1126/science.1254704

Mastrangelo, M. E., and P. Laterra. 2015. From biophysical to social-ecological trade-offs: integrating biodiversity conservation and agricultural production in the Argentine Dry Chaco. Ecology and Society 20(1):20. https:// dx.doi.org/10.5751/ES-07186-200120. Matteucci, S. D. 2012. Ecorregión Bosques Patagónicos. Pp. 489-547 in J. Morello, S. D. Matteucci, A. F. Rodríguez and M. Silva (eds.). Ecorregiones y complejos ecosistémicos argentinos. Orientación Gráfica Editora. Buenos Aires, Argentina.

Matteucci, S. D. 2012. Ecorregión Campos y Malezales. Pp. 247-263 in J. Morello, S. D. Matteucci, A. F. Rodríguez and M. Silva (eds.). Ecorregiones y complejos ecosistémicos argentinos. Orientación Gráfica Editora. Buenos Aires, Argentina.

Matteucci, S. D. 2012. Ecorregión Espinal. Pp. 349-390 in J. Morello, S. D. Matteucci, A. F. Rodríguez and M. Silva (eds.). Ecorregiones y complejos ecosistémicos argentinos. Orientación Gráfica Editora. Buenos Aires, Argentina.

Menzel, S., and J. Teng. 2010. Ecosystem services as a stakeholder】driven concept for conservation science. Conservation Biology 24(3):907-909. https://doi.org/10.1111/i.1523-1739.2009.01347.x

Meyfroidt, P., T. K. Rudel, and E. F. Lambin. 2010. Forest transitions, trade, and the global displacement of land use. Proceedings of the National Academy of Sciences 107(49):20917-20922. https://doi.org/10.1073/pnas.1014773107.

Miradi. 2017. Adaptive Management Software for Conservation Projects. URL: https://www.miradi.org/.

Morello, J., W. Pengue, and A. Rodríguez. 2005. Etapas de uso de los recursos y desmantelamiento de la biota del Chaco. Fronteras 4:1-17.

Neiff, J., and A. Poi de Neiff. 2005. Situación ambiental en la ecorregión Iberá. Pp. 177-184 en A. Brown, U. Martínez Ortiz, M. Acerbi and J. Corcuera. La Situación Ambiental Argentina 2005. Fundación Vida Silvestre Argentina, Buenos Aires.

Ojeda, R. A, C. E. Borghi, and V. G. Roig. 2002. Mamíferos de la Argentina. Biodiversidad y conservación de mamíferos neotropicales. Pp. 23-64 en G. Ceballos and J. Simonetti (eds.). Diversidad y conservación de los mamíferos neotropicales. Comisión Nacional para el Conocimiento y Uso de la Biodiversidad (México) Universidad Nacional Autónoma de México.

Olson, D. M., E. Dinerstein, E. D. Wikramanayake, N. D. Burgess, G. V. Powell, E. C. Underwood, J. A. D'Amico, I. Itoua, H. E. Strand, J. C. Morrison, C. J. Loucks, T. F. Allnutt, T. H. Ricketts, Y. Kura, J. F. Lamoreux, W. W. Wettengel, P. Hedao, and K. R. Kassem. 2001. Terrestrial Ecoregions of the World: A New Map of Life on Earth. A new global map of terrestrial ecoregions provides an innovative tool for conserving biodiversity. BioScience 51(11):933-938. https: //doi.org/10.1641/0006-3568(2001)051[0933:TEOTWA]2.0.CO;2.

Ostrom, E. 2007. Sustainable social-ecological systems: an impossibility? Available at SSRN 997834. https://doi.org/ 10.2139/ssrn.997834.

Paviolo, A., Y. E. Di Blanco, C. D. De Angelo, and M. S. Di Bitetti. 2009. Protection affects the abundance and activity patterns of pumas in the Atlantic Forest. Journal of mammalogy 90(4):926-934. https://doi.org/10.1644/08-MAMMA-128.1.

Pesaresi, M., A. Florczyk, M. Schiavina, M. Melchiorri, and L. Maffenini. 2019. GHS settlement grid, updated and refined REGIO model 2014 in application to GHS-BUILT R2018A and GHS-POP R2019A, multitemporal (1975-1990-2000-2015), R2019A. European Commission, Joint Research Centre (JRC). https:/ / doi.org/10.2905/42E8BE89-54FF-464E-BE7BBF9E64DA5218. URL: http://data.europa.eu/89h/42e8be89-54ff-464e-be7b-bf9e64da5218

Piquer-Rodríguez, M., S. Torella, G. Gavier-Pizarro, J. Volante, D. Somma, R. Ginzburg, and T. Kuemmerle. 2015. Effects of past and future land conversions on forest connectivity in the Argentine Chaco. Landscape Ecology 30(5): 817-833. https://doi.org/10.1007/s10980-014-0147-3.

Piquer-Rodríguez, M., V. Butsic, P. Gärtner, L. Macchi, L. M. Baumann, C. G. Pizarro, J. N. Volante, I. N. Gasparri, and T. Kuemmerle. 2018. Drivers of agricultural land-use change in the Argentine Pampas and Chaco regions. Applied geography 91:111-122. https://doi.org/10.1016/j.apgeog.2018.01.004.

Politi, N., M. Hunter, and L. Rivera. 2012. Assessing the effects of selective logging on birds in Neotropical piedmont and 
cloud montane forests. Biodiversity and Conservation 21(12):3131-3155. https://doi.org/10.1007/s10531-012-0358-3.

Politi, N., and L. Rivera. 2019. Limitations and advances to attain sustainable forest management in Southern Yungas of Argentina. Ecología Austral 29(01):138-145. https://doi.org/10.25260/EA.19.29.1.0.753.

Quiroga, V. A., G. I. Boaglio, A. J. Noss, and M. S. Di Bitetti. 2014. Critical population status of the jaguar Panthera onca in the Argentine Chaco: camera-trap surveys suggest recent collapse and imminent regional extinction. Oryx 48(1):141-148. https://doi.org/10.1017/S0030605312000944.

Quiroga, V. A., A. J. Noss, A. Paviolo, G. I. Boaglio, and M. S. Di Bitetti. 2016. Puma density, habitat use and conflict with humans in the Argentine Chaco. Journal for Nature Conservation 31:9-15. https://doi.org/10.1016/i.jnc.2016.02.004

Redo, D. J., H. R. Grau, T. M. Aide, and M. L. Clark. 2012. Asymmetric forest transition driven by the interaction of socioeconomic development and environmental heterogeneity in Central America. Proceedings of the National Academy of Sciences 109(23):8839-8844. https://doi.org/10.1073/pnas.1201664109.

R Team. 2018. R: A language and environment for statistical computing.

Salafsky, N., R. Margoluis, K. H. Redford, and J. G. Robinson. 2002. Improving the practice of conservation: a conceptual framework and research agenda for conservation science. Conservation Biology 16(6):1469-1479. https://doi.org/10.1046 .1523-1739.2002.01232.x

Satorre, E. 2005. Cambios tecnológicos en la agricultura argentina actual. Ciencia Hoy 15:24-31.

Sica, Y. V., R. D. Quintana, V. C. Radeloff, and G. I. Gavier-Pizarro. 2016. Wetland loss due to land use change in the Lower Paraná Riyer Delta, Argentina. Science of the Total Environment 568:967-978. https://doi.org/10.1016 i.scitotenv.2016.04.200.

Turner, B. L., E. F. Lambin, and A. Reenberg. 2007. The emergence of land change science for global environmental change and sustainability. Proceedings of the National Academy of Sciences 104(52):20666-20671. https://doi.org 10.1073/pnas.0704119104

United Nations. 2015. Transforming our world: The 2030 agenda for sustainable development. General Assembly, 70th session.

Vallejos, M., S. Aguiar, G. Baldi, M. Mastrángelo, F. Gallego, M. Pacheco-Romero, D. Alcaraz-Segura, and J. M. Paruelo. 2019. Social-Ecological Functional Types: Connecting People and Ecosystems in the Argentine Chaco. Ecosystems 23:471-482. https://doi.org/10.1007/s10021-019-00415-4

Vallejos, M., J. N. Volante, M. J. Mosciaro, L. M. Vale, M. L. Bustamante, M. L., and J. M. Paruelo. 2015. Transformation dynamics of the natural cover in the Dry Chaco ecoregion: A plot level geo-database from 1976 to 2012. Journal of Arid Environments 123:3-11. https://doi.org/10.1016/j.jaridenv.2014.11.009.

Verburg, P. H., N. Crossman, E. C. Ellis, A. Heinimann, P. Hostert, O. Mertz, and H. R. Grau. 2015. Land system science and sustainable development of the earth system: A global land project perspective. Anthropocene 12:29-41. hittps: /doi.org/10.1016/j.ancene.2015.09.004

Viglizzo, E. F., F. Lértora, A. J. Pordomingo, N. J. Bernardos, Z. E. Roberto, and H. Del Valle. 2001. Ecological lessons and applications from one century of low external-input farming in the pampas of Argentina. Agriculture, Ecosystems and Environment 83(1-2):65-81. hittps://doi.org/10.1016/S0167-8809(00)00155-9.

Viglizzo, E. F., M. F. Ricard, E. G. Jobbágy, F. C. Frank, and L. V. Carreno. 2011. Assessing the cross-scale impact of 50 years of agricultural transformation in Argentina. Field Crops Research 124(2):186-194. https://doi.org/10.1016 ifcr.2011.05.014

Villagra, P. E., and J. A. Álvarez. 2019. Determinantes ambientales y desafíos para el ordenamiento forestal sustentable en los algarrobales del Monte, Argentina. Ecología Austral 29(1):146-155. https://doi.org/10.25260/EA.19.29.1.0.752.

J. N. Volante, M. J. Mosciaro, M. C. Morales Poclava, L. M. Vale, S. A. Castrillo, J. Sawchik, G. Tiscornia, M. Fuente, I. Maldonado Ibarra, A. Vega, R. Trujillo, L. Cortéz, and J. M. Paruelo. 2015. Expansión agrícola 2000-2010 en Argentina, Bolivia, Paraguay, Uruguay y Chile. Caracterización espacial mediante series temporales de índices de vegetación. RIA, Revista de Investigaciones Agropecuarias 41(2):179-191.

Zimmerer, K. S. (ed.). 2006. Globalization and new geographies of conservation. University of Chicago Press.

Zhong, Y., C. Giri, P. S. Thenkabail, P. Teluguntla, R. G. Congalton, K. Yadav, A. J. Oliphant, J. Xiong, J. Poehnelt, and C. Smith. 2017. NASA Making Earth System Data Records for Use in Research Environments (MEaSUREs) Global Food Security-support Analysis Data (GFSAD)@30-m for South America: Cropland Extent Product (GFSAD30SACE). NASA EOSDIS Land Processes DAAC. URL: https://doi.org/10.5067/MEaSUREs/GFSAD/GFSAD30SACE.001. 